\title{
Structural Models of Self-Efficacy of Filipino Radiologic Technology Educators, Current Learners, and Prospective Students in the Senior High School
}

\author{
Mark M. Alipio * \\ * Faculty of Radiologic Technology, Davao Doctors College, Philippines \\ (e-mail: markalipiorrt@gmail.com)
}

\begin{abstract}
:
Objectives: This study aims to explore three different structural models of self-efficacy for Filipino Radiologic Technology educators, current learners, and prospective students in the senior high school.

Methodology: Subjects were 256 Radiologic Technology educators (102 males and 154 females) and 2,451 Radiologic Technology students (1,525 males and 926 females), randomly selected from 22 Radiologic Technology schools in the Philippines. A total of 4,263 prospective Radiologic Technology students from the 30 senior high schools in the Philippines were also sampled as respondents. Six instruments were used to measure help-seeking, self-esteem, social support, motivation, self-regulation, and self-efficacy. Path analysis was utilized to identify the best fitting model.

Results: The best fit models of self-efficacy for Filipino Radiologic Technology educators and learners showed that help-seeking, self-esteem, social support, motivation, and self-regulation positively influenced self-efficacy, with social support exerting the greatest causal effect. The best fit model of self-efficacy for prospective Radiologic Technology students from senior high school reported that social support completely mediates the effects of help-seeking, self-esteem, motivation, and self-regulation on self-efficacy.

Implications: The study highlights different results that could be arrived depending on whether future researchers decide to use the self-efficacy models for Filipino Radiologic Technology educators, current learners, and prospective students in the senior high school. The provision of highly supportive environment for Radiologic Technology educators, current learners, and prospective students in the senior high school is essential to increase self-efficacy, thereby improving their capabilities to hurdle the challenges in the academic milieu.
\end{abstract}

Keywords: Educators, Filipino, Help-seeking, Learners, Motivation, Path Analysis, Philippines, Radiologic Technology, Self-efficacy, Self-esteem, Self-regulation, Senior High School, Social Support, Structural Model, Teachers 


\section{INTRODUCTION}

Education in Radiologic Technology (RT) requires collaboration of teachers and learners. While the interplay of collaboration is complex and demanding, the question on how to execute courses of action required to deal with the pressure of the academic requirements is still a problem. Hence, teachers' and learners' self-efficacy are considered unprecedent factors for the success of education and schools. Teachers' efficacy is the belief or persuasion of teachers that they can influence the quality of students' learning, even of those who are considered as problematic cases or lacking motivation while learners' efficacy refers to the beliefs students hold in their capabilities to think and behave in ways that are systematically oriented toward or associated with their learning goals.

To address the concern, many researchers in the past years have tried to investigate the different antecedents of self-efficacy. Several studies reported that help-seeking, self-esteem, social support, motivation, and self-regulation were the strongest correlates of self-efficacy in both teachers and learners (Alipio, 2020; Alipio, Felizarte, Revilla, nd). However, there is a dearth of empirical evidence on the combined effect of these factors to self-efficacy. The need to identify and verify the connection among these variables is highly significant to extend the social learning theory and to provide action plans that mitigate further problems in the academe.

Meanwhile, studies pertaining to senior high school students are gaining attention in the Philippines. The adoption of $\mathrm{K}$ to 12 education of the government from neighboring and highly developed countries calls for a continuous effort to be at par with the global academic milieu. This effort requires preliminary assessment of the academic persistence of the students in order to determine the education measures needed to prevent formidable dropout scenario in the college arena. Self-efficacy score is a robust measurement of academic persistence. Previous reports showed that students reporting relatively strong self-efficacy generally achieved higher grades and were much more likely to persist in technical or scientific majors over a 1-year period than were those with low self-efficacy (Alipio, 2020). Hence, measuring senior high school students' self-efficacy and associated factors are essential input for quality assurance.

\section{OBJECTIVES OF THE STUDY}

This study aims to explore three different structural models of self-efficacy for Filipino Radiologic Technology educators, current learners, and prospective students in the senior high school. Specifically, it attempted to: (1) determine the causal relationship among helpseeking, self-esteem, social support, motivation, self-regulation, and self-efficacy, and (2) determine the best fit model of self-efficacy among the three groups.

\section{METHODOLOGY}

Sample. Subjects were 256 Radiologic Technology educators (102 males and 154 females) and 2,451 Radiologic Technology students (1,525 males and 926 females), randomly selected from 22 Radiologic Technology schools in the Philippines. A total of 4,263 prospective Radiologic Technology students from the 30 senior high schools in the Philippines were also sampled as respondents.

Instruments. Six instruments were used to collect data for the study. Help seeking was assessed using an adaptation of a 6-item help seeking attitude scale developed by Knapp and Karabenick (1991). Changes were made on the item statements and options to fit the circumstances of the study. That is, the word "tutoring" was replaced with the word "help". The answer format used was a Likert scale ranging from 1 (strongly disagree) to 6 (strongly agree). Knapp and Karabenick (1991) reported a reliability of 0.74 for the 6item scale. Rosenberg's Self-Esteem Scale (Rosenberg, 1965) was utilized to measure self-esteem. The Rosenberg Self-Esteem Scale consists of 10 items measuring the participant's global self-esteem $(\alpha=.83)$. The answer format used is a Likert scale 
ranging from 1 (strongly disagree) to 4 (strongly agree). The scale was scored such that a high score corresponds with a high level of self-esteem.

The Support Questionnaire, designed by Lozada-Santone (2001), measures perception of social support from four sources: parents, instructors, close friends, and other adults. The Support Questionnaire consists of 36 items, a total score on all sources of support is tallied using a 3 -point Likert scale, rated as $3=$ very helpful, supportive or encouraging, 2 = sometimes helpful, or supportive or encouraging or $1=$ not helpful, supportive or encouraging. The Support Questionnaire has a strong internal consistency; a Cronbach's alpha of 0.912 was reported by the developer of the scale (Lozada-Santone, 2001).

Motivation was measured by the standard open source Academic Motivation Scale (AMS) developed by Vallerand et al. (1992). The scale consists of 18 items measuring the following four sub-dimensions: intrinsic motivation, introjected regulation, identified regulation and external regulation. The answer format used was a Likert scale ranging from 1 (strongly disagree) to 5 (strongly agree). Vallerand et al. (1992) reported reliability coefficients ranging from 0.83 to 0.86 for the different subscales of the Academic Motivation Scale (AMS).

Self-regulation was measured using Self-Regulation Questionnaire (SRQ) of Brown, Miller, \& Lawendowski (1999). Reliability of the SRQ appears to be excellent. In a community sample of 83 people with varying levels of alcohol problem severity, the SRQ was administered twice, separated by 48 hours, to test stability of scores it provides (Aubrey, Brown, \& Miller, 1994). Test-retest reliability for the total SRQ score was high $(\mathrm{r}=.94, \mathrm{p}<.0001)$. Internal consistency of the scale was also quite high $(\alpha=.91)$.

The Academic Self-Efficacy Scale, as developed by Klobas, Renzi, and Nigrelli (2007) was utilized. These 10 item-scale was assessed on a seven-point Likert-type scale ranging from 1 (I am definitely not able to do this) to 7 (I am definitely able to do this). The internal consistency of this scale has been reported as 0.84 (Cronbach Alpha), which shows good internal consistency (Klobas et al., 2007).

Data Collection and Analysis. The six instruments were directly administered to the subjects with help of 30 research assistants. Path analysis was utilized to identify the best fitting model as examined by the following goodness of fit indices: Chisquare/degrees of Freedom, Root Mean Square of Error Approximation, Tucker-Lewis Index and Comparative Index. In identifying the best fitting model, all the indices must consistently fall within acceptable ranges. Chi-square/ degrees of freedom value should between $\mathrm{o}$ and 2, with its corresponding $\mathrm{p}$-value greater or equal to 0.05. Root Mean Square of Error Approximation value must be less than 0.05 and its corresponding pclose value must be greater or equal to 0.05. The other indices such as Normed Fit Index, Tucker-Lewis Index, Comparative Index and Goodness of fit index must be all greater than 0.95 . 


\section{RESULTS}

Best Fit Model of Self-Efficacy for Radiologic Technology Educators

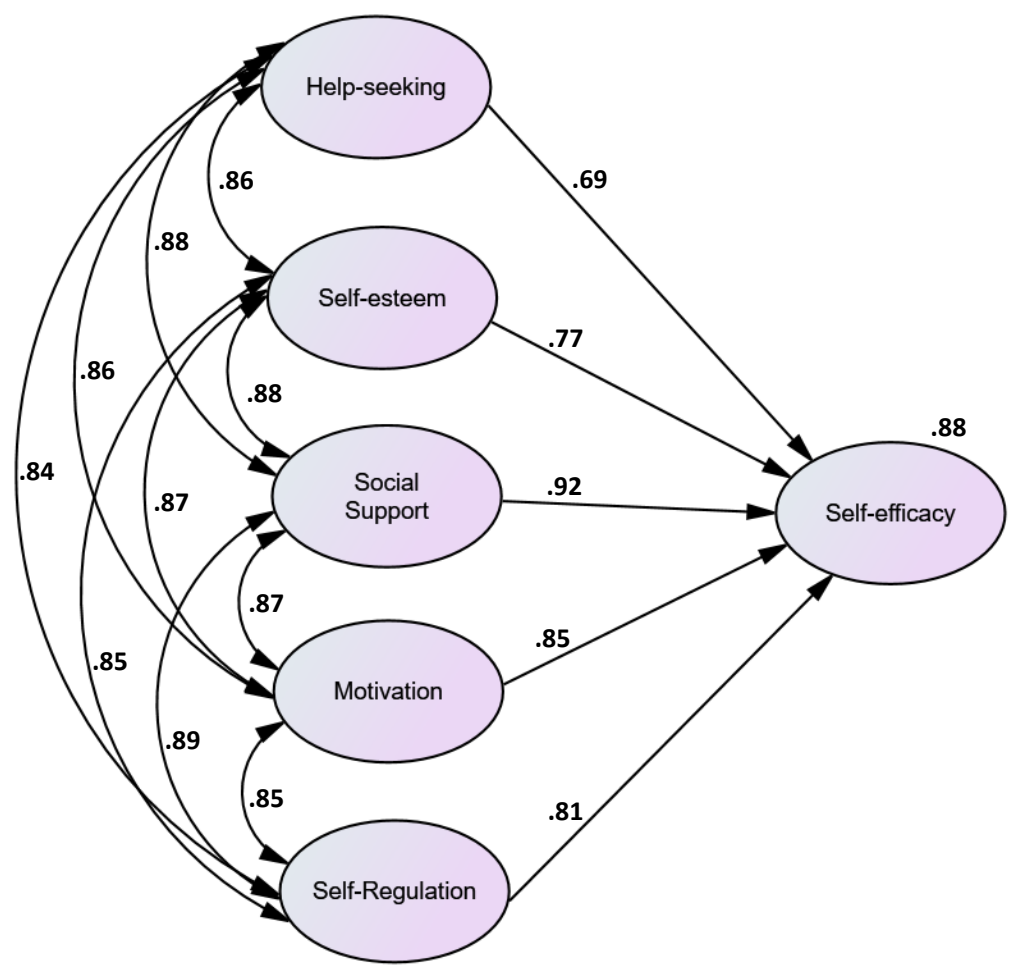

Figure 1. Best Fit Model of Self-Efficacy for Radiologic Technology Educators

The model of self-efficacy for Radiologic Technology educators depicts that help-seeking, self-esteem, social support, motivation, and self-regulation are interrelated from each other and positively influence self-efficacy (Figure 1). Preliminary correlation analysis revealed that help-seeking, self-esteem, social support, motivation, and self-regulation are positively related to self-efficacy. Approximately $88 \%$ of the variance in self-efficacy can be explained by help-seeking, self-esteem, social support, motivation, and self-regulation. The determinant with the greatest causal effect on self-efficacy was social support $(B=.92)$, followed by motivation $(\mathrm{B}=.81)$, self-regulation $(\mathrm{B}=.81)$, self-esteem $(\mathrm{B}=.77)$, and helpseeking $(\mathrm{B}=.69)$. Test for goodness of fit revealed that the model fits the data well as manifested by the following indices: $\mathrm{CMIN} / \mathrm{DF}=1.431 ; \mathrm{p}$-value $=.052 ; \mathrm{NFI}=.991$; TLI $=$ $.984 ; \mathrm{CFI}=.983 ; \mathrm{GFI}=.975 ; \mathrm{RMSEA}=.021 ;$ PCLOSE $=.088$. Chi-square value $(32.012 ; \mathrm{p}$ $=0.000$ ) was evidence of the good validity of the model. 
Best Fit Model of Self-Efficacy for Radiologic Technology Learners

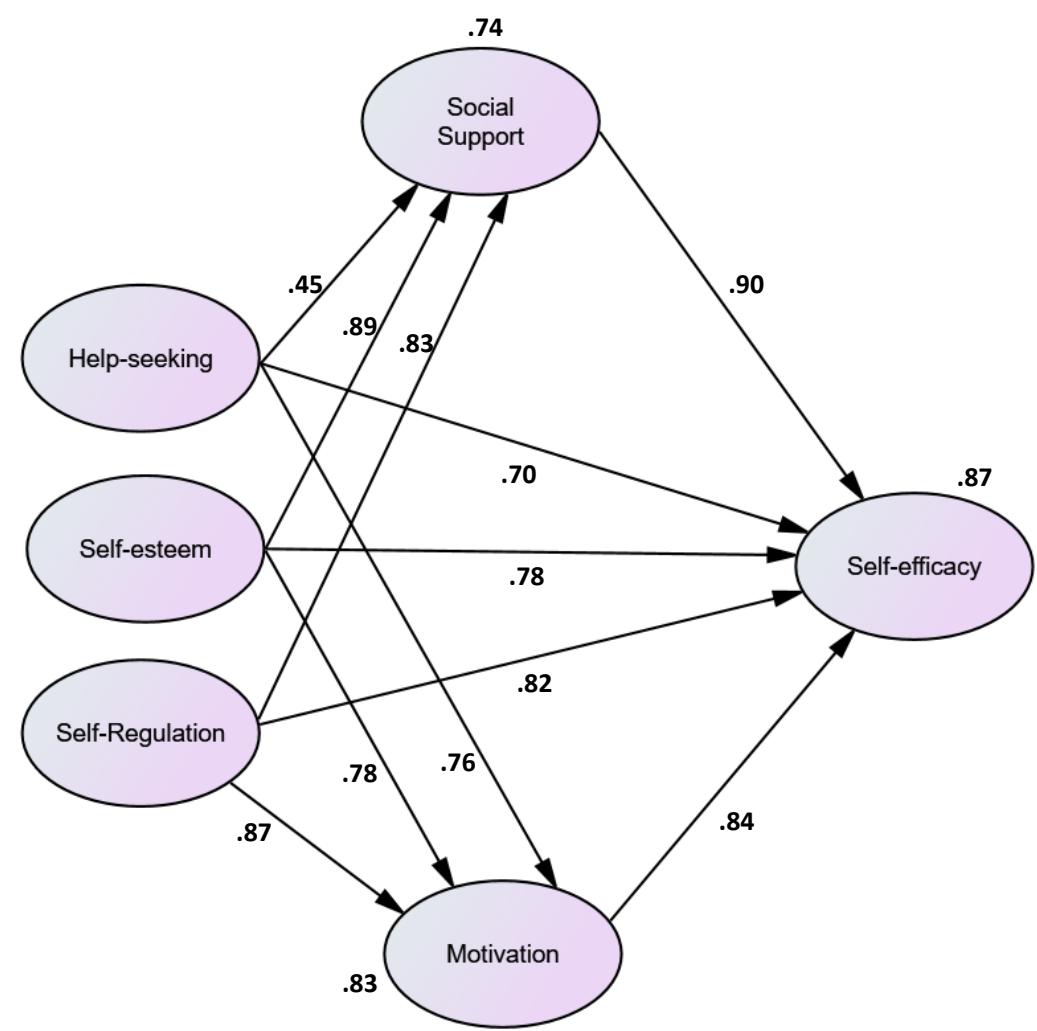

Figure 2. Best Fit Model of Self-Efficacy for Radiologic Technology Learners

The model of self-efficacy for Radiologic Technology learners depicts the positive direct influence of help-seeking, self-esteem, social support, motivation, and self-regulation on self-efficacy and several indirect paths (Figure 2). Preliminary correlation analysis revealed that help-seeking, self-esteem, social support, motivation, and self-regulation are positively related to self-efficacy. Approximately $87 \%$ of the variance in self-efficacy can be explained by help-seeking, self-esteem, social support, motivation, and self-regulation. The determinant with the greatest causal effect on self-efficacy was social support $(B=.90)$, followed by motivation $(\mathrm{B}=.84)$, self-regulation $(\mathrm{B}=.82)$, self-esteem $(\mathrm{B}=.78)$, and helpseeking $(\mathrm{B}=.7 \mathrm{O})$. Test for goodness of fit revealed that the model fits the data well as manifested by the following indices: $\mathrm{CMIN} / \mathrm{DF}=1.210$; $\mathrm{p}$-value $=.102$; NFI $=.964$; TLI $=$ $.952 ; \mathrm{CFI}=.975 ; \mathrm{GFI}=.992 ; \mathrm{RMSEA}=.031 ; \mathrm{PCLOSE}=.064$. Chi-square value $(24.325 ; \mathrm{p}$ $=0.000$ ) was evidence of the good validity of the model.

It can be observed that there are several indirect paths to self-efficacy. Help-seeking, self-esteem, and self-regulation indirectly influenced self-efficacy via social support and motivation. Sobel test was further employed to completely analyze the mediation. The test revealed that social support fully mediated the effects of help-seeking, self-esteem, and self-regulation on self-efficacy; however, motivation partially mediated the effects of help-seeking, self-esteem, and self-regulation on self-efficacy.

Two other outcome variables were presented in the path: social support and motivation. Approximately $74 \%$ of the social support variance can be explained by help-seeking, self-esteem, and self-regulation. Of the three determinants of social support, self-esteem exerted the greatest causal effect $(\mathrm{B}=.89)$, followed by self-regulation $(\mathrm{B}=.83)$, and helpseeking $(\mathrm{B}=.45)$. Approximately $83 \%$ of the motivation variance can be explained by help-seeking, self-esteem, and self-regulation. Of the three determinants of motivation, self-regulation exerted the greatest causal effect $(B=.87)$, followed by self-esteem $(\mathrm{B}=.78)$, and help-seeking $(\mathrm{B}=.76)$. 
Best Fit Model of Self-Efficacy for Prospective Radiologic Technology Students from Senior High School

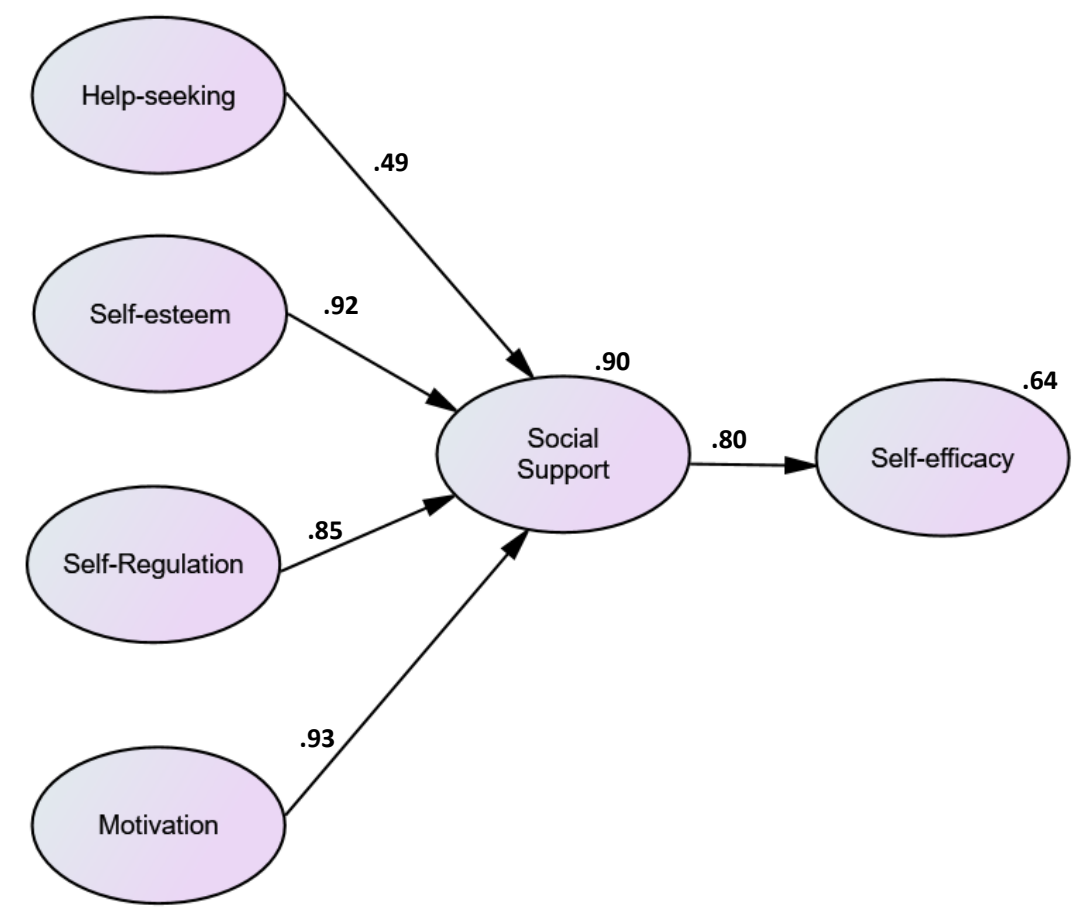

Figure 3. Best Fit Model of Self-Efficacy for Prospective Radiologic Technology Students from Senior High School

The model of self-efficacy for prospective Radiologic Technology students from senior high school depicts the positive direct influence of help-seeking, self-esteem, motivation, and self-regulation on social support, which directly influences self-efficacy (Figure 3). Preliminary correlation analysis revealed that help-seeking, self-esteem, motivation, and selfregulation are positively related to social support, which in turn is positively related to selfefficacy. Approximately $90 \%$ of the variance in social support can be explained by helpseeking, self-esteem, motivation, and self-regulation. The determinant with the greatest causal effect on social support was motivation $(B=.93)$, followed by self-esteem $(B=.92)$, selfregulation $(\mathrm{B}=.85)$, and help-seeking $(\mathrm{B}=.49)$. Meanwhile, approximately $64 \%$ of the variance in self-efficacy can be explained by social support, which exerted a positive influence on selfefficacy $(\mathrm{B}=.80)$. Sobel test revealed that social support completely mediates the effects of help-seeking, self-esteem, motivation, and self-regulation on self-efficacy. Test for goodness of fit revealed that the model fits the data well as manifested by the following indices: $\mathrm{CMIN} / \mathrm{DF}=1.341 ; \mathrm{p}$-value $=.087 ; \mathrm{NFI}=.978 ; \mathrm{TLI}=.966 ; \mathrm{CFI}=.991 ; \mathrm{GFI}=.956 ; \mathrm{RMSEA}=$ $.042 ;$ PCLOSE $=.078$. Chi-square value $(26.235 ; \mathrm{p}=0.000)$ was evidence of the good validity of the model. 


\section{DISCUSSION}

This study explored three structural models of self-efficacy for Filipino Radiologic Technology educators, current learners, and prospective students in the senior high school. In doing so, the study determined the causal relationship among help-seeking, self-esteem, social support, motivation, self-regulation, and self-efficacy, and examined the best fit model of selfefficacy among the three groups.

Based on the best fit model of self-efficacy for Filipino Radiologic Technology educators, significant causal relationships exist among help-seeking, self-esteem, social support, motivation, self-regulation, and self-efficacy. The path model showed that help-seeking, selfesteem, social support, motivation, and self-regulation are positively interrelated from each other, suggesting that an increase in the level of one variable may likely increase the level of the other (e.g. increase in help-seeking may likely increase self-esteem, social support, motivation, and self-regulation). The model also presented the significant role of helpseeking, self-esteem, social support, motivation, and self-regulation on self-efficacy of Radiologic Technology educators. Social support exerted the greatest impact on self-efficacy implying that a highly supportive environment for educators may boost their self-efficacy in teaching.

On the other hand, the best fit model of self-efficacy for Filipino Radiologic Technology learners showed that significant causal relationships exist among help-seeking, self-esteem, social support, motivation, self-regulation, and self-efficacy. Similar to the previous model, all of the variables significantly and directly influence self-efficacy; however, several indirect effects were observed in the model. For instance, help-seeking, self-esteem, and selfregulation indirectly influenced self-efficacy via social support and motivation. Sobel test was further employed to completely analyze the mediation. The test revealed that social support fully mediated the effects of help-seeking, self-esteem, and self-regulation on self-efficacy; however, motivation partially mediated the effects of help-seeking, self-esteem, and selfregulation on self-efficacy. This implies that help-seeking, self-esteem, and self-regulation will only influence self-efficacy indirectly via social support. In other words, help-seeking, self-esteem, and self-regulation will not affect self-efficacy if there is no social support. This is highly indicative of the significant role of social support among the learners of Radiologic Technology course.

Finally, the best fit model of self-efficacy for prospective Radiologic Technology students from senior high school showed that significant causal relationships exist among help-seeking, self-esteem, social support, motivation, and self-regulation. However, only social support explained self-efficacy. Help-seeking, self-esteem, motivation, and self-regulation positively and directly influence social support. It is noteworthy to mention that a full mediation by social support exists in the model. Social support completely mediates the relationship of help-seeking, self-esteem, motivation, and self-regulation on self-efficacy. This implies that help-seeking, self-esteem, motivation, and self-regulation will only influence self-efficacy indirectly via social support. In other words, help-seeking, self-esteem, motivation, and selfregulation will not affect self-efficacy if there is no social support. Similar to the best fit model of self-efficacy for Radiologic Technology learners, social support is significant in cultivating beliefs in their capabilities to think and behave in ways that are systematically oriented toward or associated with their learning goals.

The study highlights different results that could be arrived depending on whether future researchers decide to use the self-efficacy models for Filipino Radiologic Technology educators, current learners, and prospective students in the senior high school. In addition, the study showed how the variables significantly contributed to self-efficacy of the respondents. Most importantly, this study provided implications on school administrators and community in the provision of highly supportive environment for Radiologic Technology educators, current learners, and prospective students in the senior high school to improve self-efficacy, thereby increasing their capabilities to hurdle the challenges in the academic milieu. 


\section{REFERENCES}

Alipio, M. (2020). Predicting Academic Performance of College Freshmen in the Philippines using Psychological Variables and Expectancy-Value Beliefs to OutcomesBased Education: A Path Analysis.

Alipio, M. (2020). Academic Adjustment and Performance among Filipino Freshmen College Students in the Health Sciences: Does Senior High School Strand Matter?

Alipio, M. (2020). Exploring the Clinical Competencies of Radiologic Technologists in Emergency Departments of Selected Hospitals in the Philippines.

Alipio, M. M., Felizarte, L. P., \& Revilla, D. L. PSYCHOSOCIAL FACTORS, EXPECTANCY-VALUE BELIEFS TO OUTCOME-BASED EDUCATION APPROACH AND ACADEMIC PERFORMANCE AMONG FIRST YEAR STUDENTS.

Alipio, M. M., Felizarte, L. P., \& Revilla, D. L. Outcomes-Based Education Approach Correlates and Academic Performance of College Radiologic Technology Freshmen.

Florentino, C. B., Gustilo, R. L., Jailani, H. U., Maceren, M. R., Periabras, N. O., \& Alipio, M. M. The Clinical Competencies of Radiologic Technology Interns of Batch 2018-2019 on Radiological Science Modalities: Basis for Proposed Enhancement Program. 